\title{
Elements of genetic counseling for inborn errors of metabolism
}

\author{
Natalie Beck* and Carolyn Applegate \\ Department of Genetic Medicine, Johns Hopkins University School of Medicine, Baltimore, MD, USA
}

\begin{abstract}
Individually rare but collectively common, inborn errors of metabolism (IEMs) are genetic disorders of metabolic pathways that result in multisystem dysfunction and may reduce lifespan if untreated. The IEM patient population requires collaborative care from primary care providers such as pediatricians/general practitioners, specialists, metabolic geneticists, dietitians and genetic counselors. Ideally, a patient and his or her family would have regular access to a comprehensive care team, however, some patients may require their primary care provider to manage some aspects of their IEM-related care. There have been many advances in the diagnosis and treatment of IEMs especially since the initiation of newborn screening in the United States for phenylketonuria (PKU) in the 1960s. These advancements have resulted in individuals with IEMs living into adulthood and having children of their own, creating an aging patient population and subsequent needs across this increased lifespan. Genetic counseling is a key component in the care and education of patients with IEMs at the time of the initial diagnosis and beyond. We highlight many genetic counseling concepts and issues that need to be addressed and reassessed over the lifespan of a patient with an IEM. This review is intended to support the patient's primary care provider in recognizing these concepts and issues to reinforce for patients with IEMs in the medical home and the importance of referring for genetic counseling.
\end{abstract}

Keywords: Genetic counseling, genetic counselor, inborn errors of metabolism, metabolic, recessive, recurrence, newborn screening, carrier testing, coping, guilt, shame, transition

\section{Introduction}

Genetic counseling is the process of helping people understand and adapt to the medical, psychological and familial implications of genetic contributions to disease [1]. The first class of genetic counselors graduated in 1971 from Sarah Lawrence College, however, core concepts of genetic counseling have been an element of medical care since the recognition of the familial nature of human disease [1]. Genetic counselors are healthcare professionals with specialized graduate degrees and experience in the areas of both medical genetics and psychosocial counseling. Genetic counselors work as members of a healthcare team, providing risk assessment, education and support to individuals and families at risk for, or diagnosed with, genetic conditions. These responsibilities encompass interpreting and disclosing genetic testing results, providing supportive counseling, and acting as patient advocates [1]. Currently, there are over 5,000 genetic counselors certified by the American Board of Genetic Counseling (ABGC), and this number continues to grow [2].

Inborn errors of metabolism (IEMs) are a subset of genetic conditions characterized by a block in a metabolic pathway due to deficient or absent activity of an enzyme [3]. IEMs can be split into three categories: small molecule diseases, large complex molecule diseases, and others [4]. This discussion

\footnotetext{
*Corresponding author: Natalie Beck, MGC, CGC, Department of Genetic Medicine, Johns Hopkins University School of Medicine, 600 N. Wolfe Street, Blalock 1008, Baltimore, MD 21287, USA. Tel.: +1 410955 3071; Fax: +1 4106149246 ; E-mail: nbeck1@jhmi.edu.
} 
focuses on small molecule diseases as they comprise the core of the metabolic newborn screening program in the United States and have specific treatment recommendations, which are mainly dietary interventions, although many concepts here could apply to the wider IEM population. The diagnosis of a small molecule IEM in the newborn period presents an array of medical and psychosocial challenges that continue over the lifespan. Genetic counselors are uniquely poised to address many of these needs of patients and their families. Ideally, every patient and family receiving a new diagnosis of an IEM would meet with a genetic counselor at the time of the new diagnosis and periodically over time. However, we recognize that primary care providers serve as the patient's medical home and, therefore, may field questions about the condition, medical family history, and additional issues. Primary care providers should recognize when a referral to a genetic counselor would be beneficial to the patient and family (see Table 1). The goal of this review is to provide primary care providers with information about the medical and psychosocial issues commonly experienced by patients with IEM and their families to allow for ongoing support outside of the genetics clinic.

\section{Genetic concepts}

\section{A. Inheritance Patterns \& Family History}

IEMs are by definition genetic conditions and are generally single gene disorders though additional genetic and environmental factors affect the natural history. IEMs follow the principles of Mendelian genetics and follow autosomal recessive, autosomal dominant, X-linked or mitochondrial patterns of inheritance. Family history is a powerful tool for identifying and estimating risk for medical conditions within a family, particularly via a three-generation pedigree. Each type of inheritance follows a specific pattern that can often be identified through pedigree assessment and allows for recurrence risk to be estimated for each family member.

Most IEMs identified by newborn screening are inherited in an autosomal recessive pattern [5]. Recessive conditions are recognizable on a pedigree when the patient is affected and both parents are unaffected (no vertical transmission) [5]. When more than one person in the family has the condition, they are usually full siblings. Other family members, however, may be carriers, meaning that they have a pathogenic variant in one of their two copies of the gene causing that IEM. Carriers have an increased risk to have a child with the condition compared to the general population. The majority of patients and their parents with recessive IEMs will have no family history of the condition. Genetic counselors are experts in obtaining family history, identifying patterns of inheritance, and communicating this information to families. Referral to a genetic counselor is indicated when a new diagnosis is made and at any point when the family has questions about these concepts, so that a full family history can be obtained, assessed, and discussed with the family to promote their understanding (see Table 1).

\section{B. Recurrence Risks}

The chance for a disease to recur in a family is determined by the mechanism of inheritance for that particular gene and condition. In the case of autosomal recessive conditions, unaffected carrier parents of an affected child have a $25 \%$ chance in each pregnancy to have another affected child as shown in Fig. 1. A patient with a recessive IEM will pass on one pathogenic variant to each of his/her children. Thus, all of his/her children will have at least one pathogenic variant and be unaffected carriers. The chance for each child to be affected would be determined by the carrier status of the patient's partner. Genetic counseling is recommended for patients with IEMs and their partners prior to and during the family planning process to allow for informative carrier testing based on the diagnosis (see Table 1).

The chance for other family members to have a child with the recessive IEM depends on their carrier status. Calculation of exact risk is possible using disease incidence and degree of relationship to affected individual(s) in the family. Practically though, many individuals' decisions to have or forgo 
N. Beck and C. Applegate / Elements of genetic counseling for inborn errors of metabolism

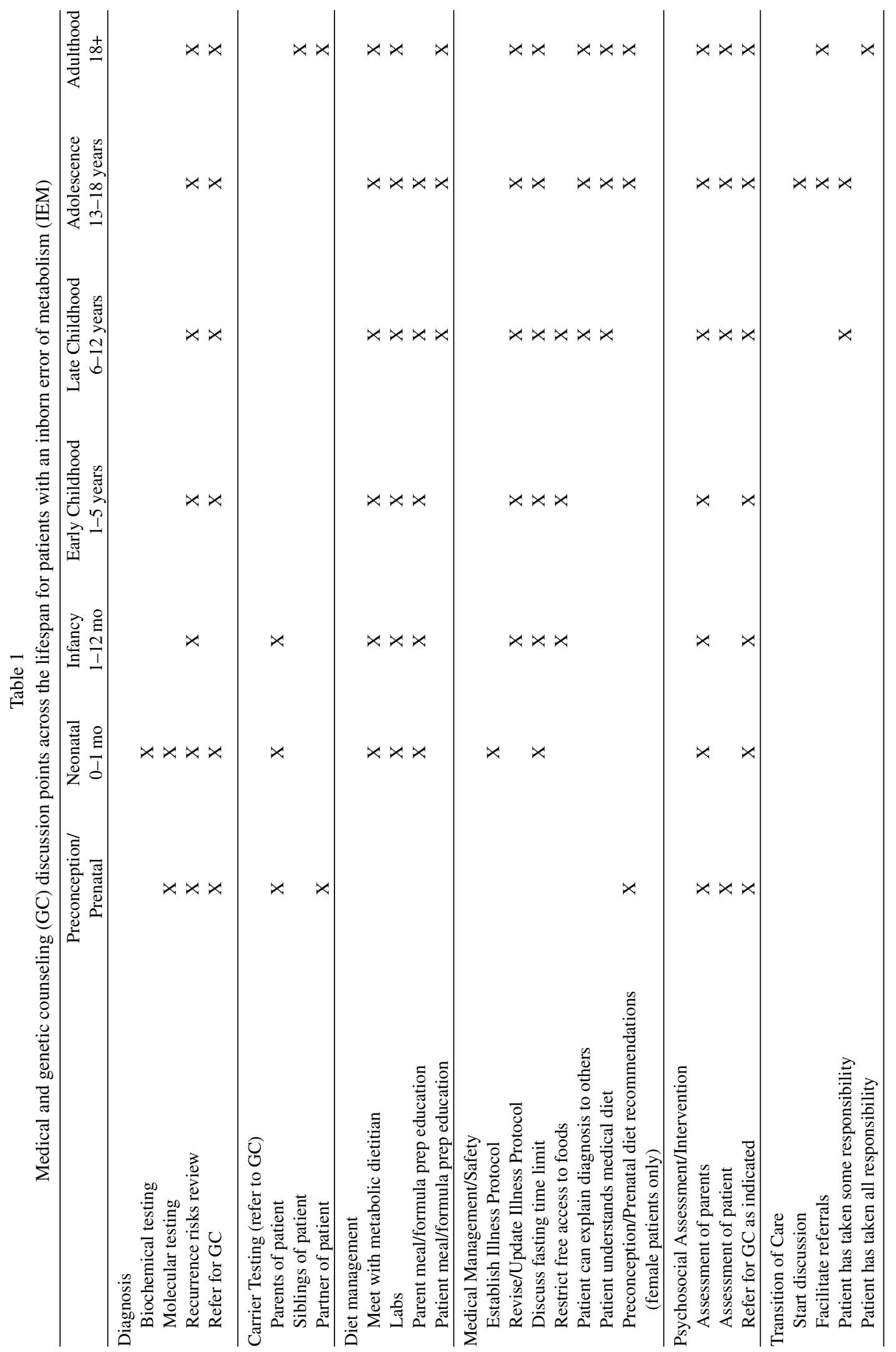




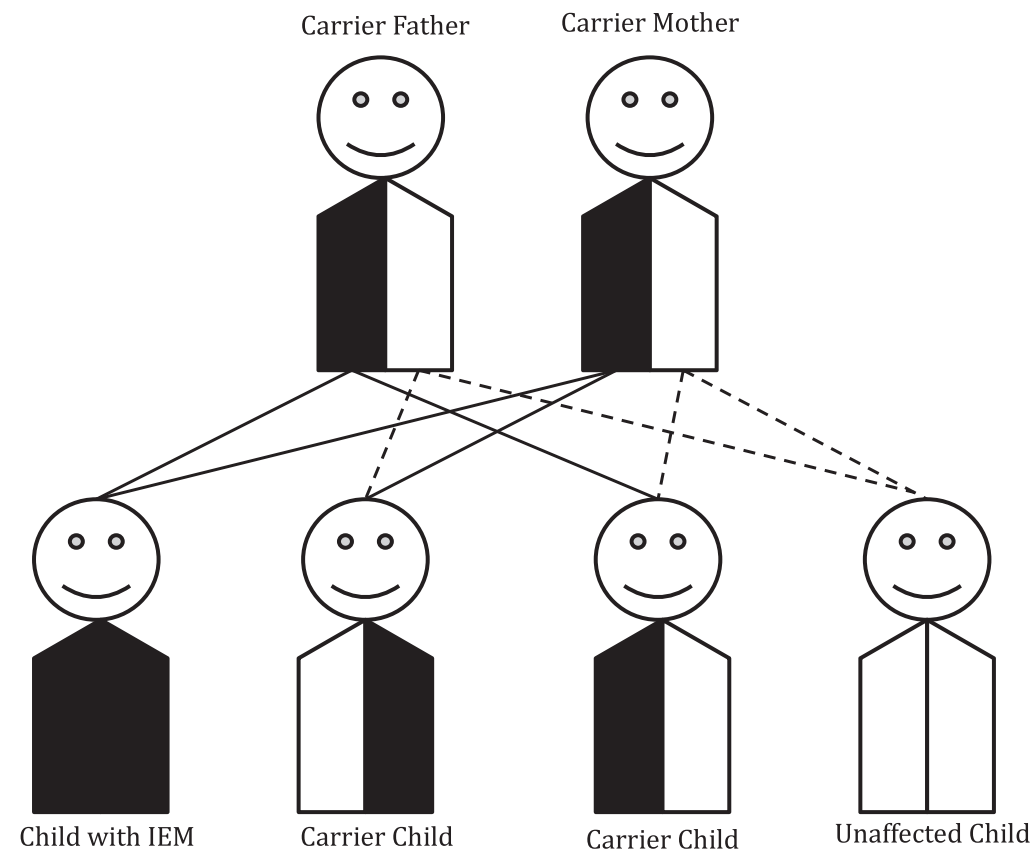

Fig. 1. Autosomal recessive inheritance. Most inborn errors of metabolism (IEMs) are inherited in an autosomal recessive pattern. In this case, parents of a child with an IEM will have a 1/4 (25\%) chance with each pregnancy of having another child with that IEM. Unaffected siblings have a $2 / 3$ chance of being a carrier.

carrier testing and prenatal diagnosis are not significantly influenced by differences of $0.1 \%$ (i.e. a risk of 1 in 200 versus a risk of 1 in 1000).

In consanguineous families, the chance for a relative of a patient with an IEM to be a carrier is often higher than that estimated based on the degree of relationship due to the fact that consanguinity is often present in multiple generations. In these cases, it's appropriate to offer carrier screening to anyone in the family, regardless of degree of relationship. Individuals who are not known to be consanguineous but who are from small populations isolates, whether geographical or cultural in origin, may also have a higher than the calculated risk to be a carrier or have an affected child. Special consideration should be taken in these circumstances with regard to carrier testing, in particular, recognizing that published carrier frequencies and disease prevalence may not accurately reflect the incidence in that specific population. Genetic counselors have expertise in calculating risk, identifying at-risk family members and facilitating communication of this information with families.

\section{Carrier Identification}

Ideally, carrier testing should occur prior to achieving a pregnancy to allow an individual or couple to fully understand their options regarding carrier detection and/or prenatal diagnosis. Performing carrier testing prior to a pregnancy also gives individuals and couples the option of preimplantation genetic testing for monogenic disorders (PGT-M). Testing to determine carrier status of minors should be approached thoughtfully, as to preserve the child's autonomy in choosing whether he or she would like to know this information [6]. Identification of carrier status in minors may occur when a sibling is undergoing diagnostic testing for a suspected IEM or when carrier status is identified incidentally by newborn screening [7]. In this case, it's important to review with the parents, and with the siblings of the patient in an age-appropriate manner, the chances for each to have an affected child in the future.

Within the last few years, expanded carrier screening for many genetic conditions, including many IEMs, has become much more prevalent. Expanded carrier screening offers an unbiased approach to 
identification of carriers by extending the same test platform to all, regardless of ethnicity [8]. Because most individuals with autosomal recessive disease have no family history of that particular disorder, expanded carrier screening allows for the identification of couples who have no family history of a disease, but have a $25 \%$ chance of having a child with an autosomal recessive condition (such as an IEM). As these individuals typically have no personal experience with the condition for which they are identified to be a carrier, they often need more information about the disease itself and its impact on health and life, which genetic counselors can provide. Not all IEMs are included on expanded screening panels, and the breadth of gene variants included for analysis and reporting by the testing laboratory can vary, so for couples pursuing carrier screening who have a relative with a known IEM, it is imperative to confirm whether the known familial variant(s) would or would not have been detected by the universal screening panel. Due to these challenges, targeted testing of known familial variants is the optimal way to identify carriers in families with affected individuals, even if this in addition to an expanded carrier panel. When patients and their families are considering carrier testing, they should be referred to a genetic counselor to ensure that the appropriate test for that patient or family member is performed based on his or her specific risk and that they are informed of all of their testing options and potential outcomes of testing.

\section{Reproductive Options}

Another service provided to patients and families by genetic counselors is to review reproductive and family planning options and facilitate a discussion regarding the benefits, risks, and limitations of these options in the context of their risk perception, medical status, and personal/cultural beliefs [1]. Genetic counselors can help families navigate all the available options for future family planning after having a child with IEM. These discussions should include all options available to that family which may include: natural conception with or without prenatal diagnosis, preconception diagnosis (specifically preimplantation genetic testing aka PGT-M), use of one or more donor gametes and/or surrogacy, adoption, and the option of foregoing having additional children.

In order to perform prenatal genetic diagnosis or PGT-M, the specific gene and pathogenic variants causing the patient's IEM must be known. Prenatal diagnosis is performed during an ongoing pregnancy by chorionic villus sampling (CVS) at approximately 11-13 weeks gestation or amniocentesis at approximately 16-20 weeks. The advantage of prenatal diagnosis is that testing is highly accurate with very low false positive and false negative rates; however, individuals must weigh this with a risk of procedure-related miscarriage, generally quoted as about 1/300 [7]. Families may pursue prenatal diagnosis to prepare for having an affected child, including deciding on location of delivery and neonatal care plan, or, may choose the available option to terminate an affected pregnancy. PGT-M is performed on one or more cells removed by the lab after in vitro fertilization (IVF) has occurred in order to identify unaffected embryos which can then be transferred for pregnancy [7]. The advantage of PGT-M is that testing is highly accurate, though it is still recommended that prenatal diagnosis be performed for confirmation after pregnancy is achieved. High cost and access to these technologies can be a barrier for some families. Additionally, IVF introduces an increased risk for other health conditions, such as imprinting disorders [9]. These issues are often best explored with a genetic counselor who can discuss advantages and disadvantages of all options as well as facilitate decisionmaking.

\section{Psychosocial considerations}

In addition to the medical genetics and molecular test interpretation expertise that genetic counselors can provide to families and the larger care team, genetic counselors additionally have expertise in psychosocial assessment and counseling techniques [10]. The psychosocial domain includes the emotional, 
cognitive, familial, social, economic and cultural beliefs of those involved [10]. A new medical diagnosis, particularly one in a child, often affects all of these areas of the psychosocial domain [11, 12]. Utilizing genetic counselors for psychosocial assessment and counseling is recommended, but all medical providers working with families who have a new IEM diagnosis would benefit from anticipating and recognizing specific interventions and developmental landmarks where psychosocial issues may be more prominent and affect the family and the patient's care (see Table 1). We will expand on these as well as additional chronic psychosocial issues present for patients and families with IEMs below.

\section{A. Grieving the Diagnosis}

In the United States, many patients are diagnosed with an IEM as part of the metabolic newborn screening program, leading to a new diagnosis in an infant who is typically only a few days or weeks old. For many families there is an initial period of grief that surrounds a new diagnosis and the 'loss of the expected child.' This type of grief goes beyond the parents' immediate concerns for the child's health and is a more profound psychological process. A new diagnosis forces the parents to confront their expected or imagined roles of parenthood, family and how they view the child as an extension of themselves [10]. The grief and psychological disruption of the envisioned roles/processes described above occurs when a diagnosis of a rare and serious medical problem is made and can be exacerbated when a family is not appropriately informed about newborn screening and possible results.

We encourage all care providers involved in prenatal, neonatal, and pediatric care realms to continue to educate families about the newborn screening program in unbiased ways, sharing the purpose of newborn screening and that screening tests will include both false and true positives as part of the larger goal of screening infants for numerous conditions that require immediate implementation of medical treatment(s) to prevent complications, but are not detectable by prenatal testing, screening or ultrasounds. It is helpful for providers to call the test by its appropriate name such as 'metabolic newborn screening' instead of outdated terms such as 'the PKU test' to prevent confusion to families who may misunderstand what the screening test includes. The Advisory Committee on Heritable Disorders in Newborns and Children (ACHDNC) issues a Recommended Universal Screening Panel (RUSP) and as of July 2018, the RUSP guidelines includes 35 core conditions and 26 secondary conditions (exact number of screened conditions varies state by state) [13]. For primary care providers who are often the first to contact a family about abnormal newborn screen results, it is essential that they assess how the family responds to the information to help guide them for prompt follow up. A decrease in distress has been shown for parents of children with true and false positives from metabolic newborn screening when the caregivers were connected with a metabolic center/expert providers to support them at the time of the initial screen results [14].

When families are grieving the loss of the expected child in the setting of a new diagnosis, reactions can include any or all of the reactions on the traditional grief spectrum including shock, denial, anger, guilt, shame, despair/grief, and sadness. Over time there is typically acceptance although every parent goes through this process in their own time and parents may go back into a state of grief at various times in the child's life $[1,10]$. Families who have a child with an IEM (or other rare disease) experience considerable losses across the lifespan with impacts in the emotional, social, practical and global domains $[11,12]$. Parents and family caregivers may grieve in different ways and so supporting each caregiver based on their needs will help the entire family system function. For those parents/caregivers who become stuck in this grief state and are unable to progress towards resolution/acceptance, it is recommended that the parent/family be referred for ongoing family therapy with a licensed psychologist or family therapist. Both the genetic counselor and the primary care provider should continue to assess parents and caregivers who are in the grief process to identify those who may benefit from additional therapeutic counseling services. 


\section{B. Guilt and Shame}

Additional chronic psychosocial issues that occur in families that have a child or children with an IEM are guilt/shame issues related to carrier status and the burden of disease on the patient and the family. While they can be elicited from the same or similar experiences, guilt and shame are interrelated but unique psychological reactions. Both are normal responses to adverse events over which a person has little or no control and a patient or parents' willingness to discuss their feelings of guilt and shame is often culturally influenced. Guilt implies a sense of responsibility, suggesting that the individual could have changed the outcome. Guilt is a common psychological defense mechanism against powerful feelings of helplessness and in the face of what may be perceived as a 'random event.' Feelings of shame, while potentially felt simultaneously to guilt, arise from the person's perception of external judgement of them, their family, or their choices $[10,15]$. The parents of a child with an IEM often experience feelings of guilt and shame, particularly when learning of their obligate carrier status (recessive disorders). This situation can be further challenging to the family dynamic for the few IEMs inherited in an X-linked manner in which only the mother can be the obligate carrier for the condition in the child.

In addition to psychosocial assessment, the genetic counselor is trained to provide context and support for the parents during expressions of guilt/shame by providing normalizing statements. Some examples of these that a general practitioner could reinforce include: 'We are all carriers for numerous rare conditions'; 'Carriers are nearly always healthy and unaffected, you would not have known you were a carrier.' Genetic counselors also provide medical genetic context for the etiology of the child's condition. For many parents, understanding the cause of their child's condition can reduce the feelings of guilt and shame [10], and so genetic counseling at the time of diagnosis but additionally over time can help parents learn this new and often complex information (see Table 1).

There may be other times when guilt or shame is experienced by the patient or family related to an IEM, requiring ongoing reassessment [15]. For example, the child may have shame related to the requirement for a strict medical diet, particularly among peers [16]. Another common scenario can be that the unaffected siblings may experience a form of 'survivor guilt' since they did not inherit the same IEM. The parents of the child and/or unaffected siblings (or other family members) may feel shame if they opt for prenatal diagnostic testing due to concerns about the heritability of the rare IEM in a future child. This is often particularly challenging for the parents who already have one child with an IEM who experience conflicting feelings about prenatal diagnosis with the goal of a pregnancy resulting in a future child without that IEM, and the love they have for their existing child with an IEM. Regular follow up with genetics providers including genetic counselors is recommended to allow ongoing assessment for coping and the need for additional resources or referrals.

\section{Diet Management: Food is a Prescription}

Many of the psychosocial stressors for a patient with an IEM and parents of a child with an IEM often relate to the major alterations required for nutrition [15-17] which often includes careful calculations about foods and daily caloric intake closely scrutinized based on a medical diet prescription, which contrasts greatly from how most people without an IEM view food and meal planning.

One particular early stressor can start in the neonatal period for some families as breastfeeding may have to be interrupted or stopped altogether based on some IEM diagnoses. This is only done when necessary for the health of the patient, but can be additionally traumatic to the parents and how they envisioned early parenthood, breastfeeding, nutrition and bonding would occur with their child.

For many patients with an IEM, their diet is a carefully calculated prescription and foods need to be portioned out, weighed, measured and/or mixed in an exact manner [15-19]. Based on the IEM diagnosis, some patients may need to incorporate a medical formula and/or medical foods that have been altered specifically to their IEM diagnosis to allow for good metabolic control. This can be challenging 
for many families day to day but additionally stressful in times of holidays or other celebrations when extra calories are consumed by those without an IEM and certain foods and dishes may be central to those events. Additional challenges for the patient with IEM may be times of fasting related to their religious practices, which can be dangerous to some persons with IEMs. Lastly, providers should educate parents and caregivers of the importance of celebrating and rewarding the child with an IEM with non-food types of treats/praise.

The providers and metabolic dietitian caring for a child with an IEM should make every effort to customize the patient's prescribed diet in order to incorporate foods that are familiar/preferred by the family to increase compliance. It is also crucial that there is ongoing education to the patient and family about the medical diet as this has been found to increase patient autonomy and independence as well as diet compliance as the patient ages [15-17, 19]. Metabolic foods and formulas are not consistently covered by health insurance plans, despite their necessity for the health of the child, so medical providers should be prepared to submit pre-authorization requests and letters of medical necessity for the patient at the time of diagnosis and regularly as needed.

An additional component of stress can be the frequency of changes to the medical diet for the child as he/she grows (See Table 1). As the child progresses to adding purees and then solid foods, the family of the child with an IEM will need ongoing support from a nutritionist/dietitian with training in metabolic genetics for guidance $[12,15-17,19]$. The ratio of protein to carbohydrates to fats and any restricted foods will evolve based on the child's growth velocity, activity level and their metabolic state based on serial labs. Parents and caregivers are often encouraged to have diet journals to help them document the child's intake day to day. As children increase the time that they are eating meals away from the home at school, daycare, camp, etc., caregivers will need to develop a plan with the child to assess intake.

\section{Managing Common Illnesses in the Patient with an IEM}

Across the developmental spectrum, there are additional times and scenarios we can anticipate dietary requirements and restrictions to become additionally challenging, such as during times of illnesses when intake may be decreased. For the general provider supporting a child and family with an IEM, common illnesses and issues in childhood such as febrile/viral illnesses, the common cold, and feeding-related issues for children (i.e., frequent spit-ups and typical food refusals) can be lifethreatening in those with an IEM and require prompt medical intervention. Therefore, these seemingly common childhood events can become large stressors for the caregiver of a child with an IEM and it can be helpful to understand these issues in order to help support these families.

Chronic spit-ups and episodes of vomiting are common in childhood due to reflux and intercurrent illnesses, but for some patients with IEMs can also be symptoms of poor metabolic control. Further challenging for caregivers of patients with an IEM is that these children frequently experience metabolic decompensation during routine illnesses [18]. Some patients are not allowed to fast for long periods, making times of illness with decreased intake more challenging. Patients with IEMs often do not tolerate illnesses well due to the increased metabolic stress and the alteration of intake (typically less) and fasting (typically more) during times of illness [18]. For the parent/caregiver at home, navigating the correct reaction to an episode of vomiting or recurrent spit ups can be stressful and may need assistance from the child's primary care provider and specialized medical care team for management. Many patients will require prompt intervention of IV hydration with dextrose and possibly other medications to help maintain stability and prevent metabolic crisis until the patient's regular metabolic diet can be reintroduced; however, specific management should be determined by a metabolic specialist for each patient.

We recommend that the family work with their specialty medical care team and primary care provider to develop a written 'Emergency Illness Protocol.' This should be customized to the patient's IEM 
diagnosis, age, and other aspects of their health. It should include the immediate/urgent steps any provider in an emergency department should take to assess and stabilize the patient (stat labs before IV, IV fluids prescription, additional supplements/medications needed, and other recommendations) as well as emergency contact information for the child's metabolic care team. This protocol should be updated as the child ages and their treatment changes. This protocol should be on file with the child's medical providers, school, camps, coaches, religious group leader, and any other supervising adult that cares for the child and of course the parents/caregivers should have copies. Having an emergency illness protocol prepared and on file helps families feel more prepared and in control of an otherwise stressful situation and can help healthcare providers unfamiliar to the child/IEM diagnosis provide prompt and effective care. The genetic counselor can provide anticipatory guidance to families about these expected childhood illnesses/topics and help advocate for them within their care team by helping the parents to explore resources and concepts to be included in their child's illness management plan that the family understands and they are able to implement (See Table 1).

\section{E. IEMs can be Isolating}

While many providers have a main focus on the health of the child with an IEM, their attention to the wellness of the larger family is also crucial to assess and support. Many families who have a child with a rare or ultra-rare genetic diagnosis will highlight their feelings of isolation related to this diagnosis. Caring for a child with a rare disease can be socially isolating and can impact the psychological health of the caregiver, their long term employability, and other domains of living [11, $12,15,17,18,20]$. Parents are often forced into a position of 'being the expert' for their child when encountering a new healthcare provider, teacher, coach or other caregiver for the child, a role that many parents and caregivers are unprepared to fill. Parents may also experience frustration since their infant/child with a rare IEM has a typical appearance ('they don't look sick'), but their medical course and dietary needs are anything but typical. The child's primary care provider can help to serve as a medical home to the family and a direct conduit to the child's metabolic team in order to help support the family and facilitate smooth communication about the child's care plan.

Genetic counselors are available to connect the parents of the child with an IEM to quality resources and social connections relating to this diagnosis. This process can be additionally provided and reinforced by the child's primary care provider. In addition to the STAR-G and Baby's First Test websites relating to newborn screening, there are often support groups that are diagnosis-specific. Over the past few years, families are now locating each other through social media (e.g. Facebook) and online support group webpages which have both open and closed groups for numerous genetic conditions. As these are rarely proctored by a provider experienced with the IEM of focus, both genetic and primary care providers should remind families that support groups are not intended to provide medical advice, but instead should afford the family a place of community and a platform to share experiences. Any potential learned alternative treatment approaches gleaned from a support group should be discussed with the child's medical team including the metabolic geneticist/nutritionist in the context of that child's needs prior to changes in management.

Not every parent/caregiver is ready to engage with others in a support group at the time of diagnosis, so it is helpful for the child's providers to regularly assess a family's use of support groups and the potential advantages and challenges with these groups with the child and family. If families become interested in this option in the future, this can also be an excellent time to refer the family back for genetic counseling to review additional aspects related to the diagnosis and assess coping (see Table 1). Facilitating the connection for parents of a child with an IEM to others will decrease feelings of isolation and help normalize many of the family's experiences with their child's IEM diagnosis [1, 15]. Connecting families also often leads to networking, diagnosis-based conferences and conventions and allows for patients to connect with one another and to connect with specialty providers who 
may attend those meetings, decreasing stigma and shame that the patient may feel in their immediate community where they are likely to be the only person with their IEM diagnosis.

\section{F. Rebellion}

While it can be challenging for families to maintain diet compliance, this process is further complicated during periods of normal rebellion and autonomy development such as in toddlerhood, preadolescence and adolescence. In these times of normal development when the child is learning to express preferences and test boundaries in an effort to increase independence, families may see increased struggle to adhere to the metabolic diet prescription [15-19, 21]. Anticipating these times of challenge allows caregivers to brainstorm appropriate compromises and solutions. Empowering children to responsibly become a partner in food selections with their caregivers and slowly take on additional aspects of their diet management is important to help foster self-esteem and normal childhood developmental independence $[15,16,19]$. For the adolescent patient, there should be additional discussions relating to medical transition and for the patient to begin taking more ownership and responsibility for his/her diet and intake, unless the patient has cognitive impairment that prohibits this level of independence $[15,16,18,19,21]$.

\section{G. Transition to Adulthood}

As treatments for IEMs have become more advanced, the lifespan of these patients has also increased. Adolescents with IEMs should receive updated genetic counseling about their diagnosis and their own recurrence risks as they approach adulthood (see Table 1). This is important to introduce prior to the individual reaching the stage of active family planning, as patients may only know the recurrence risks provided to their parents, which are different from their own (see Section 2B). The primary care provider should refer the adolescent patient with an IEM for genetic counseling for these discussions to allow them information to help empower their healthcare choices and increase self-esteem in adulthood and also help to facilitate the medical transition process [15-19, 21].

While many patients live into adulthood, some may not be able to live independently due to cognitive impairments or other complex medical needs. It is important for the caregivers of these patients to obtain guardianship (or other legal arrangement) based on the needs of the patient to help determine long term needs and support systems. These patients will likely benefit from additional government-based resources in adulthood.

\section{H. Burden of Disease}

Patients with IEMs and their parents/caregivers have expressed experiencing a high burden of disease $[11,12,15,18,20]$. An IEM diagnosis impacts diet, lifestyle, intercurrent illness management and multi-systemic function for many patients. Additionally, for some patients with IEMs even with lifelong dietary compliance there can be cognitive and psychological impacts of the disease (e.g., executive functioning deficits or ADHD) [16, 17]. For females with an IEM, there is an additional burden of disease that can be felt prior to and throughout a pregnancy when additional attention is required for strict metabolic control.

It is important to acknowledge the burden of disease for patients and families and support their efforts in managing their own/their child's diagnosis. Acknowledging the family's feelings about their child's IEM can help them cope with the diagnosis and opens up communication channels should the caregivers become overwhelmed and need additional support.

\section{Conclusion}

Patients and families receiving a diagnosis of a rare genetic condition frequently experience many normal psychosocial reactions that include grief, guilt, shame, isolation, frustration, and psychologi- 
cal challenges such as anxiety and depression relating to chronic management of a condition with a high disease burden. Genetic counselors help patients and their families understand and adapt to the medical, psychological and familial implications of genetic contributions to disease and provide ongoing psychosocial assessment and counseling throughout the lifespan of the patient. Specifically for patients with IEMs, we highlighted the psychosocial concepts which genetic counselors are assessing and addressing that primary care providers should also be aware of to reinforce for families, to be able to anticipate challenges, prepare for periods of transition, and help the patient and the family cope with the unique challenges of living with a rare condition through resource and support identification. Genetic counselors also help families understand the medical genetic components of the IEM diagnosis including molecular test results, recurrence risks, and carrier test recommendations, timing, and coordination. Some families receiving a new diagnosis of an IEM in their child may have easy access to a genetic counselor while others may only see this type of provider sparingly. Primary care providers (general practitioners and pediatricians) are frequently the providers most familiar and trusted by the patient and family, making them a crucial resource of information. Primary care providers can use this resource to guide their discussions with their patients and families impacted by IEMs, to reinforce the information provided to a family by a genetic counselor, and can additionally utilize this resource to help identify times when referral back for additional genetic counseling would be beneficial to the patient and family.

\section{References}

[1] W.R. Uhlmann, J.L. Schuette and B.M. Yashar, editors: The Guide to Genetic Counseling, 2nd ed, New Jersey, Wiley-Blackwell, 2009.

[2] National Society of Genetic Counselors, Professional Status Survey 2019: Executive Summary, https://www.nsgc. org/page/whoaregeneticcounselors.

[3] A.E. Garrod, Inborn Errors of Metabolism, London, Oxford University Press, 1923.

[4] E. Gilbert-Barness and P.M. Farrell, Approach to diagnosis of metabolic diseases, Translational Science of Rare Diseases 1 (2016), 3-22.

[5] C.R. Scriver, A.L. Beaudet, W.S. Sly and D. Valle, editors: The Metabolic and Molecular Bases of Inherited Disease, 7th ed, New York, McGraw-Hill, 1995.

[6] Canadian Pediatric Society [CPS], Guidelines for genetic testing of health children, position statement (B 2003-01), Paediatric Child Health 8(1) (2003), 42-45.

[7] L.P. Shulmana and P.M. Farrell, Translational research advances a new era of prenatal diagnosis and newborn screening, Translational Science of Rare Diseases 3 (2018), 55-82.

[8] J.G. Edwards, G. Feldman, J. Goldberg, A.R. Gregg, M.E. Norton, N.C. Rose, A. Schneider, K. Stoll, R. Wapner and M.S. Watson, Expanded carrier screening in reproductive medicine-points to consider: A joint statement of the American College of Medical Genetics and Genomics, American College of Obstetricians and Gynecologists, National Society of Genetic Counselors, Perinatal Quality Foundation, and Society for Maternal-Fetal Medicine, Obstet Gynecol 3 (2015), 653-662.

[9] G. Lazaraviciute, M. Kauser, S. Bhattacharya, P. Haggarty and S. Bhattacharya, A systematic review and meta-analysis of DNA methylation levels and imprinting disorders in children conceived by IVF/ICSI compared with children conceived spontaneously, Hum Reprod Update 21 (2015), 555-557.

[10] J. Weil, Psychosocial Genetic Counseling, New York, Oxford, 2000.

[11] C. von der Lippe, P.S. Diesen and K.B. Feragen, Living with a rare disorder: A systematic review of the qualitative literature, Mol Genet Genomic Med 5 (2017), 758-773.

[12] H. Campbell, R.H. Singh, E. Hall and N. Ali, Caregiver quality of life with tyrosinemia type 1, J Genet Couns 3 (2018), 723-731.

[13] The Advisory Committee on Heritable Disorders in Newborns and Children (ACHDNC), Newborn Screening: Towards a Uniform Screening Panel and System, https://www.hrsa.gov/advisory-committees/heritable-disorders/rusp/ index.html. 
[14] K. O’Connor, T. Jukes, S. Goobie, J. DiRaimo, G. Moran, B.K. Potter, P. Chakraborty, C.A. Rupar, S. Gannavarapu and C. Prasad, Psychosocial impact on mothers receiving expanded newborn screening results, Eur J Hum Genet 26 (2018), 477-484.

[15] S.L. Weber, S. Segal and W. Packman, Inborn Errors of Metabolism: Psychosocial challenges and proposed family systems model of intervention, Mol Genet Metab 105 (2012), 537-541.

[16] E. Witalis, B. Mikoluc, R. Motkowski, J. Sawicka-Powierza, A. Chrobot, B. Didycz, A. Lange, R. Mozrzymas, A. Milanowski, M. Nowacka, M. Piotrowska-Depta, H. Romanowska, E. Starosteck, J. Wierzba, M. Skorniewska, B.I. Wojcicka-Bartlomiejczyk, M. Gizewsk and H. Car, Polish Society of Phenylketonuria, Phenylketonuria patients' and their parents' knowledge and attitudes to the daily diet - multi-centre study, Nutr Metab 14 (2017), 57.

[17] A.M. Bosch, A. Burlina, A. Cunningham, E. Bettiol, F. Moreau-Stucker, E. Koledova, K. Benmedjahed and A. Regnault, Assessment of the impact of phenylketonuria and its treatment on quality of life of patients and parents from seven European countries, Orphanet J Rare Dis 10 (2015), 80.

[18] M.T. Abi-Wardé, C. Roda, J.B. Arnoux, A. Servais, F. Habarou, A. Brassier, C. Pontoizeau, V. Barbier, M. Bayart, V. Leboeuf, B. Chadefaux-Vekemans, S. Dubois, M. Assoun, C. Belloche, J.M. Alili, M.C. Husson, F. Lesage, L. Dupic, B. Theuil, C. Ottolenghi and P. de Lonlay, Long-term metabolic follow-up and clinical outcome of 35 patients with maple syrup urine disease, J Inherit Metab Dis 40 (2017), 783-792.

[19] C. Cazzorla, G. Bensi, G. Biasucci, V. Leuzzi, F. Manti, A. Musumeci, F. Papadia, V. Stoppioni, A. Tummolo, M. Vendemiale, G. Polo and A. Burlina, Living with phenylketonuria in adulthood: The PKU ATTITUDE study. Mol Genet Metab Rep 16 (2018), 39-45.

[20] A. Gelrud, K.R. Williams, A. Hsieh, A.R. Gwosdow, A. Gilstrap and A. Brown, The burden of familial chylomicronemia syndrome from the patients' perspective, Expert Rev Cardiovasc Ther 15 (2017), 879-887.

[21] G.M. Enns and W. Packman, The adolescent with an inborn error of metabolism: Medical issues and transition to adulthood, Adolesc Med 13 (2002), 315-329. 